\title{
A Web Page Classifier Library Based on Random Image Content Analysis Using Deep Learning
}

\author{
Leonardo Espinosa Leal \\ Arcada UAS \\ Helsinki, Finland \\ leonardo.espinosaleal@arcada.fi \\ Amaury Lendasse \\ The University of Iowa \\ Iowa City, Iowa, US
}

\author{
Kaj-Mikael Björk \\ Arcada UAS \\ Helsinki, Finland \\ kaj-mikael.bjork@arcada.fi \\ Anton Akusok \\ Arcada UAS \\ Helsinki, Finland
}

\begin{abstract}
In this paper we present a methodology and the corresponding Python library ${ }^{1}$ for the classification of webpages. Our method retrieves a fixed number of images from a given webpage, and based on them classifies the webpage into a set of established classes with a given probability. The library trains a random forest model build upon the features extracted from images by a pre-trained deep network. The implementation is tested by recognizing weapon class webpages in a curated list of 3859 websites. The results show that the best method of classifying a webpage into the studies classes is to assign the class according to the maximum probability of any image belonging to this (weapon) class being above the threshold, across all the retrieved images. Further research explores the possibilities for the developed methodology to also apply in image classification for healthcare applications.
\end{abstract}

\section{CCS CONCEPTS}

- Computing methodologies $\rightarrow$ Computer vision; Machine learning algorithms; • Software and its engineering $\rightarrow$ Software libraries and repositories; • Human-centered computing $\rightarrow$ User interface management systems; • Applied computing $\rightarrow$ Health care information systems;

\section{KEYWORDS}

webpage classification, computer vision, deep learning

ACM Reference Format:

Leonardo Espinosa Leal, Kaj-Mikael Björk, Amaury Lendasse, and Anton Akusok. 2018. A Web Page Classifier Library Based on Random Image Content Analysis Using Deep Learning. In Proceedings of ACM Woodstock conference (PETRA'18). ACM, New York, NY, USA, Article 4, 4 pages. https: //doi.org/10.475/123_4

$\overline{{ }^{1} \text { https://bitbucket.org/akusok/wibc }}$

Permission to make digital or hard copies of part or all of this work for personal or classroom use is granted without fee provided that copies are not made or distributed for profit or commercial advantage and that copies bear this notice and the full citation on the first page. Copyrights for third-party components of this work must be honored.

For all other uses, contact the owner/author(s).

PETRA'18, June 26, Corfu, Greece

(C) 2018 Copyright held by the owner/author(s).

ACM ISBN 123-4567-24-567/08/06 ..\$15.00

https://doi.org/10.475/123_4

\section{INTRODUCTION}

Web classification is a hard but necessary task for different reasons: security, social conventions, parent control among others[2,17]. It is also important for internet searchers because accurate classification can drive to better services. In the recent times a new application has emerged: Automating classification of healthcare information on the web [7,11], were the main goal is to help users to avoid, filter or ranking web content during treatment of chronic diseases or improvement of habits for the treatment of internet addictions [6, 10].

Traditional methods such as naive-Bayes or $k$-nearest neighbours have shown a good performance for the classification of web content [15]. However, the fast grown of the internet webpages mainly towards complex and interactive visual content makes the performance of these methods much poorer. In general, most of the techniques for the classification of webpages are based in the analysis of on-page features such as url name, textual content [13] and tags [15] and more rarely visual content $[4,8]$. With the rise of deep learning as the method for image classification due to its accuracy, almost to human level, and the everyday more powerful computational resources in basic workstations have motivated the study of the visual features as a suitable source of information for classification of internet content.

In this work we classified webpages using the content images by means of a pre-trained neural network. The links of the images from a given webpage are obtained and then randomly shuffled. Then, a defined associated subset of all images is downloaded and then classified by our algorithm: A random forest model build upon the features of the pre-trained network. The whole process is carry out in memory. The final outcome is a list of probabilities linked to each image. These values are related to the pre-trained classes, which in our case is related with the weapon content. The presented method is highly accurate and can be easily extended to a large number of classes.

\section{METHODOLOGY}

The Website Image-Based Classification (WIBC) library written in Python 3.6 implements the novel functionality. It retrieves images from a given url and then calculates probabilities of belonging to a particular class for these images. Current study focuses on one relevant class: Weapons, for which a large corpus of training and test data is available from the currently running CloSer project, but 
the methodology is extensible to any number of arbitrary classes. The deep learning back-end runs on an open source ${ }^{2}$ MXNet [5] library.

The whole url content of a webpage is accessed using the requests python package. Links to all image content are extracted using the lassie python package ${ }^{3}$ from the url, and optionally from all possible sub-urls. Then image formatting function fetches images from these links and sets them in RGB format, dropping corrupted, inaccessible or tiny images. A search for image objects and their formatting continues iteratively until a required number of valid images is obtained. Such approach reduces url analysis latency, because not all links are followed. Valid images are returned as python image objects in a list, without a need for drive storage.

A parallel code implementation is written using a ProcessPool job (from the Pebble python package ${ }^{4}$ ). Distributed jobs are created incrementally as more image links are discovered, and the process pool terminates immediately after obtaining a necessary number of valid images. Parallel implementation does not support suburl search, because it does not run with incremental image link discovery that greatly reduces a single url processing time.

In the next step, the associated features of each image are extracted using a deep network. Here we used a pre-trained ${ }^{5}$ deep neural network formally known as Full ImageNet Network aka Inception21k. This model is a pre-trained model on full imagenet dataset [9] with 14,197,087 images in 21,841 classes. The model is trained by only random crop and mirror augmentation. The network is based on Inception-BN network [12]. The final outcome is a numpy array of dimension: $21,841 \times$ number of images.

The 21,841 class predictions of the pre-trained model are used as inputs for our second stage model. It is trained on own set of images: a sanitized dump of random 120,342 images, and a set of 16,358 images from weapon websites (with a mix of weapon content and random content). A pure set of 793 manually sorted images of weapon content is used for testing. The class probability predictions are done by Random Forest Classifier from the Scikitlearn library [14]. Therefore, in the last step, the matrix of extracted features is turned into weapon class probabilities by means of this model.

The WIBC library works by instantiating a WIBC_weapon object, that loads a deep learning model and a random forest classifier into its memory. Then the run(url) function fetches images from a website, and returns probabilities of them to belong to the Weapon category. The WIBC_weapon settings include the maximum number of images to download per website, and whether to use GPU acceleration (with Nvidia CUDA 7.5 or 8.0).

\subsection{Methodology Under Development}

There are three directions of development for the nearest future. The first one extracts the upper-layer features from a deep network. These features provide universal and robust image description, that

\footnotetext{
${ }^{2}$ Python package https://pypi.python.org/pypi/mxnet, accelerated version https://pypi.python.org/pypi/mxnet-cu80mkl ${ }^{3} \mathrm{http}: / /$ lassie.readthedocs.io

${ }^{4}$ https://pebble.readthedocs.io

${ }^{5}$ https://github.com/dmlc/mxnet-model-gallery/blob/master/ imagenet-21k-inception.md
}

is independent of object classes and needs no re-training. The original network computes its classes from them with logistic regression. The proposed solution is to train a regularized linear model from these features towards our classes of interest. Then re-training a model on new dataset would involve only re-training the linear model, that is very fast.

The second approach is to store already observed images from each website (in some robust representation), and check all new images against them. Web images are highly repetitive across webpages. Knowing that an image was observed on a website of a particular class provides valuable classification information, may be faster than a deep network, and works with any hard-to-classify image content like Cults website.

The third approach is to gather more training data from thirdparty image datasets. This involves running the WIBC library on large image datasets like Flickr1M (1 million images) to find probably relevant images to a particular category, and then manually filtering those images to extract the relevant ones. Another way to increase the training dataset are image rotation and mirroring.

Machine learning in health-care applications are a field that has been studied, but that also have great opportunities for further development. In Björk et al. 2016 [3], the outline of the Huntington disease prediction project was presented, whereas the actual missing value imputation framework for more accurate prediction of the Huntington disease was presented by Akusok et al. [1].

\section{EXPERIMENTAL RESULTS}

We tested our implementation on a curated list of webpages ${ }^{6}$. The list includes a collection of 1006 websites with content related to weapons, 1010 to alcohol, 715 to dating, 999 to shopping and 504 to random internet content (such as nasa.gov, google.com, etc). We did not have access, in average, to $9 \%$ of the webpages mainly due to web crawling protection or non-existing domain.

The results for the list of weapon related webpages is presented in the Fig.1. The results were plotted in ascending order, depending of the mean value of the obtained probabilities of each webpage (see figure). We have analyzed a random subset of maximum ten images per webpage. The algorithm assigns a probability from 0 to 1 of belonging to a weapons class (from white to blue in the color scale). In average the result for a given webpage takes around 10 seconds, however this time can be tune depending of the user configuration (timeout, connection requests) and the internet speed connection. The download process can be done in parallel depending of the capabilities of the machine and for extracting the features of the images, a gpu option is included.

After collecting the results for all the webpages, the question to solve is what would be the criteria to decide if a webpage belongs to the weapons class or not? We have proposed two methods, first using the mean value of the probabilities assuming that for a given webpage these follow a Gaussian distribution, then we say if it belongs to the weapons class for a given threshold value. The Second method is classify the webpage if at least a $n$ number ${ }^{7}$ of the obtained images belong to the weapons class for a given threshold.

\footnotetext{
${ }^{6} \mathrm{~F}-$ Secure, private communication

${ }^{7} n$ can be $1,2,3, \ldots$ or 10 ., the maximum number of retrieved images.
} 




Figure 1: Result for a curated list of webpages with weapons content in ascending order. We retrieved a maximum of ten images (when is possible) and assigned a probability of belonging to a weapons class. The mean value, assuming a Gaussian distribution of probabilities, is plotted on the right side of the main graph.

In the Figs. 2 and 3 appear plotted the Precision-Recall and ROC curves for the two methods. The curves for the first method is plotted in magenta-squares meanwhile the for the second method, for each value of $n$, the result is plotted with colored circles.

Following the plots, it is clear that the best method, with the best Precision-Recall or less false positive rate consists, is the second proposed method with $n=1$. The respective balance point (where Sensitivity $=$ Specificity) is equal to 0.81 and it is obtained when the probability threshold is around 0.41 . The second best result is obtained with the second method at $n=2$. The first method is ranked third.

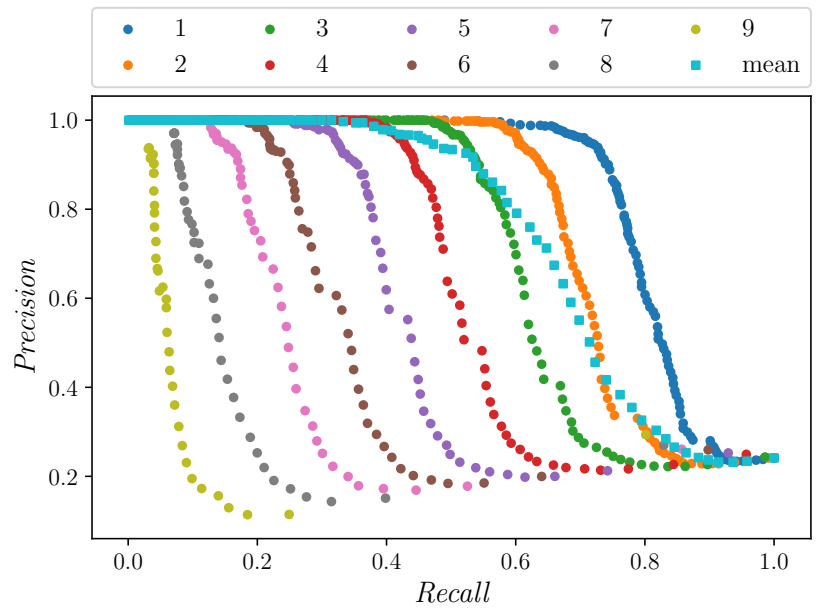

Figure 2: Precision-Recall curves for the different methods of measuring the presence of weapons in the studied websites. The first method is presented in magenta-squares and the second method for different values of $n$, from 1 to 9 is presented in colored circles.

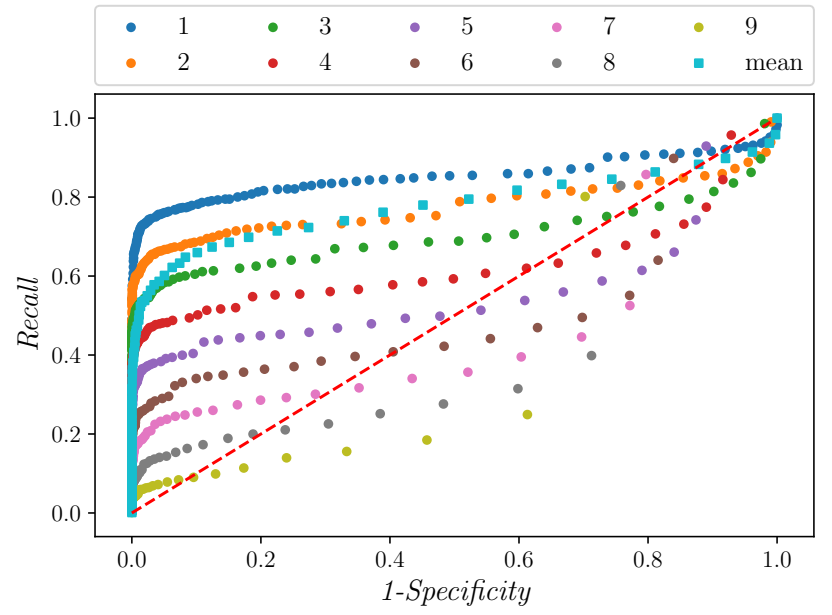

Figure 3: ROC curves for the different methods for measuring the presence of weapons in the studied websites. The first method is presented in magenta-squares and the second method for different values of $n$, from 1 to 9 is presented in colored circles.

\section{CONCLUSION AND FURTHER RESEARCH}

In the classification of webpages, the analysis of the content of images is the best criteria so far. We propose that it is possible to classify a webpage in the weapons class by a single randomly selected image from the webpage belonging to this class. The threshold for this probability can be balanced depending of the precision or less false positive rate criteria.

Two highlights of the proposed method are high accuracy in image content analysis, and an extreme simplicity of implementation. 
The most complex part of image feature extraction is performed by a state-of-the-art deep learning model pre-trained on millions of images, that currently can be obtained with a single line of code ${ }^{8}$. This saves weeks of development time on model structure selection, training parameter tuning and the training itself. Random Forest is a robust classifier that learns in seconds from arbitrary input features. The simplicity of implementation allows for flexible applications of the proposed method to any problem domain, and for easy scalability.

Further research includes exploration of the proposed method for classification of images in the health-care sector. In general, Machine learning techniques are very valuable in the health-care domain (e.g. the example of missing value imputation in [1, 3]) In this case the possible application areas may be different from the Huntington disease prediction. As discussed, there are lots of diagnostic images processed in the health-care system like brain tumor detection [16]. In addition presumptive health-care processes, like filtering the content for people who try to quit smoking (for instance) can be of extreme value.

\section{ACKNOWLEDGMENT}

This material is based on work jointly supported by the Tekes project Cloud-assisted Security Services (CloSer). The authors would like to thank their collaborators from F-Secure for sharing of the scientific research data.

\section{REFERENCES}

[1] Anton Akusok, Emil Eirola, Kaj-Mikael Björk, Yoan Miche, Hans Johnson, and Amaury Lendasse. 2017. Brute-force Missing Data Extreme Learning Machine for Predicting Huntington's Disease. In Proceedings of the 10th International Conference on PErvasive Technologies Related to Assistive Environments (PETRA '17). ACM, New York, NY, USA, 189-192.

[2] Farman Ali, Pervez Khan, Kashif Riaz, Daehan Kwak, Tamer Abuhmed, Daeyoung Park, and Kyung Sup Kwak. 2017. A Fuzzy Ontology and SVM-Based Web Content Classification System. IEEE Access 5 (2017), 25781-25797.

[3] Kaj-Mikael Björk, Emil Eirola, Yoan Miche, and Amaury Lendasse. 2016. A New Application of Machine Learning in Health Care. In Proceedings of the 9th ACM International Conference on PErvasive Technologies Related to Assistive Environments (PETRA '16). ACM, New York, NY, USA, Article 49, 4 pages.

[4] R. Burget and I. Rudolfova. 2009. Web Page Element Classification Based on Visual Features. In 2009 First Asian Conference on Intelligent Information and Database Systems. 67-72. https://doi.org/10.1109/ACIIDS.2009.71

[5] Tianqi Chen, Mu Li, Yutian Li, Min Lin, Naiyan Wang, Minjie Wang, Tianjun Xiao, Bing Xu, Chiyuan Zhang, and Zheng Zhang. 2015. MXNet: A Flexible and Efficient Machine Learning Library for Heterogeneous Distributed Systems. CoRR abs/1512.01274 (2015)

[6] Steven S. Coughlin, Lovoria B. Williams, and Christos Hatzigeorgiou. 2017. A systematic review of studies of web portals for patients with diabetes mellitus. mHealth 3, 6 (2017)

[7] Robert M. Cronin, Daniel Fabbri, Joshua C. Denny, and Gretchen Purcell Jackson. 2015. Automated Classification of Consumer Health Information Needs in Patient Portal Messages. AMIA Annu Symp Proc 2015 (05 Nov 2015), 1861-1870. 2245873[PII].

[8] Viktor de Boer, MaartenW. van Someren, and Tiberiu Lupascu. 2011. Web Page Classification Using Image Analysis Features. Springer Berlin Heidelberg, Berlin, Heidelberg, 272-285.

[9] J. Deng, W. Dong, R. Socher, L. J. Li, Kai Li, and Li Fei-Fei. 2009. ImageNet: A large-scale hierarchical image database. In 2009 IEEE Conference on Computer Vision and Pattern Recognition. 248-255.

[10] Mark Griffiths. 2001. Sex on the internet: Observations and implications for internet sex addiction. The fournal of Sex Research 38, 4 (2001), 333-342.

[11] Sachio Hirokawa and Emi Ishita. 2014. Non-topical classification of healthcare information on the web. Frontiers in Artificial Intelligence and Applications, Vol. 262. IOS Press, Netherlands, 237-247.
[12] Sergey Ioffe and Christian Szegedy. 2015. Batch Normalization: Accelerating Deep Network Training by Reducing Internal Covariate Shift. In Proceedings of the 32nd International Conference on Machine Learning (Proceedings of Machine Learning Research), Francis Bach and David Blei (Eds.), Vol. 37. PMLR, Lille, France, 448-456.

[13] Martin Milicka and Radek Burget. 2013. Web document description based on ontologies. In Informatics and Applications (ICIA), 2013 Second International Conference on. IEEE, 288-293.

[14] Fabian Pedregosa, Gaël Varoquaux, Alexandre Gramfort, Vincent Michel, Bertrand Thirion, Olivier Grisel, Mathieu Blondel, Peter Prettenhofer, Ron Weiss, Vincent Dubourg, et al. 2011. Scikit-learn: Machine learning in Python. Fournal of Machine Learning Research 12, Oct (2011), 2825-2830.

[15] Xiaoguang Qi and Brian D. Davison. 2009. Web Page Classification: Features and Algorithms. ACM Comput. Surv. 41, 2, Article 12 (Feb. 2009), 31 pages.

[16] Maite Termenon, Manuel Graña, Alexandre Savio, Anton Akusok, Yoan Miche, Kaj-Mikael Björk, and Amaury Lendasse. 2016. Brain MRI Morphological Patterns Extraction Tool Based on Extreme Learning Machine and Majority Vote Classification. Neurocomputing 174, Part A (2016), 344 - 351. https: //doi.org/10.1016/j.neucom.2015.03.111

[17] Marco Vanetti, Elisabetta Binaghi, Barbara Carminati, Moreno Carullo, and Elena Ferrari. 2011. Content-Based Filtering in On-Line Social Networks. Springer Berlin Heidelberg, Berlin, Heidelberg, 127-140.

\footnotetext{
${ }^{8}$ https://keras.io/applications
} 